\title{
Ghostscript 9.21 Color Management
}

\author{
Michael J. Vrhel, Ph.D. \\ Artifex Software \\ 7 Mt. Lassen Drive, A-134 \\ San Rafael, CA 94903, USA \\ www.artifex.com
}

\begin{abstract}
This document provides information about the color architecture in Ghostscript 9.21. The document is suitable for users who wish to obtain accurate color with their output device as well as for developers who wish to customize Ghostscript to achieve a higher level of control and/or interface with a different color management module.
\end{abstract}

Revision 1.6

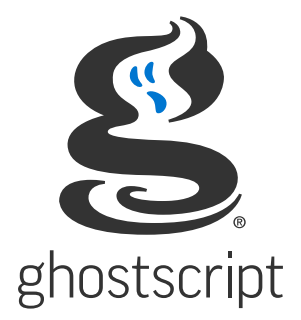




\section{Introduction}

With release 9.0, the color architecture of Ghostscript was updated to primarily use the ICC[1] format for its color management needs. Prior to this release, Ghostscript's color architecture was based heavily upon PostScript[2] Color Management (PCM). This is due to the fact that Ghostscript was designed prior to the ICC format and likely even before there was much thought about digital color management. At that point in time, color management was very much an art with someone adjusting controls to achieve the proper output color.

Today, almost all print color management is performed using ICC profiles as opposed to PCM. This fact along with the desire to create a faster, more flexible design was the motivation for the color architectural changes in release 9.0. Since 9.0, several new features and capabilities have been added. As of the 9.21 release, features of the color architecture include:

- Easy to interface different CMMs (Color Management Modules) with Ghostscript.

- ALL color spaces are defined in terms of ICC profiles.

- Linked transformations and internally generated profiles are cached.

- Easily accessed manager for ICC profiles.

- Easy to specify default profiles for source DeviceGray, DeviceRGB and DeviceCMYK color spaces.

- Devices can readily communicate their ICC profiles and have their ICC profiles set.

- Operates efficiently in a multithreaded environment.

- Handles named colors (spots) with ICC named color profile or proprietary format.

- ICC color management of Device-N colors or alternatively customizable spot color handing.

- Includes object type (e.g. image, graphic, text), rendering intent and black point compensation into the computation of the linked transform.

- Ability to override document embedded ICC profiles with Ghostscript's default ICC profiles.

- Easy to specify unique source ICC profiles to use with graphic, image and text objects.

- Easy to specify unique destination ICC profiles to use with graphic, image and text objects. 
- Easy to specify different rendering intents (perceptual, colorimetric, saturation, absolute colorimetric) for graphic, image and text objects.

- Easy to specify different black point compensation settings for graphic, image and text objects.

- Ability to make use of a PDF output intent ICC profile.

- Ability to use an NCLR ICC output profile when rendering to a separation device.

- Control to force gray source colors to black ink only when rendering to output devices that support black ink.

- Ability to make use of device link ICC profiles for direct mapping of source colors to the device color space.

- Ability to make use of device link ICC profiles for retargeting from SWOP/Fogra standard color space to a specific device color space.

- Ability to monitor for the presence of color on individual pages, which is useful for certain print systems.

- Ability to specify different default transparency blending color spaces.

- Ability to specify a post rendering ICC profile for certain devices.

The document is organized to first provide a high level overview of the architecture. This is followed by details of the various functions and structures, which include the information necessary to interface other color management modules to Ghostscript as well as how to interface specialized color handling operations.

\section{Overall Architecture and Typical Flow}

Figure 1 provides a graphical overview of the various components that make up the architecture. The primary components are:

- The ICC manager, which maintains the various default profiles.

- The link cache, which stores recently used linked transforms.

- The profile cache, which stores internally generated ICC profiles created from PostScript CIE based color spaces and CalRGB, CalGray PDF color spaces. 
- The profiles contained in the root folder iccprofiles, which are used as default color spaces for the output device and for undefined source colors in the document.

- The color management module (CMM), which is the engine that provides and performs the transformations (e.g. little CMS).

- The profiles associated with the device, which include profiles dependent upon object type, a proofing profile and a device link profile.

In the typical flow, when a thread is ready to transform a buffer of data, it will request a linked transform from the link cache. When requesting a link, it is necessary to provide information to the CMM, which consists of a source color space, a destination color space, an object state (e.g. text, graphic, or image), black point compensation setting and a rendering type (e.g. perceptual, saturation, colorimetric). The linked transform provides a mapping directly from the source color space to the destination color space. If a linked transform for these settings does not already exist in the link cache, a linked transform from the CMM will be obtained (assuming there is sufficient memory - if there is not sufficient memory then the requesting thread will need to wait). Depending upon the CMM, it is possible that the CMM may create a lazy linked object (i.e. create the real thing when it is asked to transform data). At some point, a linked transform will be returned to the requesting thread. The thread can then use this mapping to transform buffers of data through calls through an interface to the external CMM. Once the thread has completed its use of the link transform, it will notify the link cache. The link cache will then be able to release the link when it needs additional cache space due to other link requests.

\section{PDL Color Definitions and ICC Profiles}

To help reduce confusion, it is worthwhile to clarify terminology. In particular, the use of the terms process color and device color need to be defined in the context of ICC profiles. Both PDF [3] and PostScript (PS) have a distinction between process colors and device colors. In $\mathrm{PS}$, there is a conversion (e.g. via UCR/BG) from device colors to process colors. In an ICC work flow, the colors are transformed directly from an input color space (often called the source space) to an output color space (often called the destination space). The output color space defined by the device's ICC profile is a mapping to what PDF and PS define as the process color space of the device. In other words, the "device color space" as defined by the device's ICC profile IS the process color space of PDF and PS. The ICC profile of the device is a mapping from a CIE color space to the process color space AND from the process color space to a CIE color space.

To understand this better, it may help to understand the method by which a print based ICC profile is created. To create an ICC profile for a device, a chart is printed using its process 


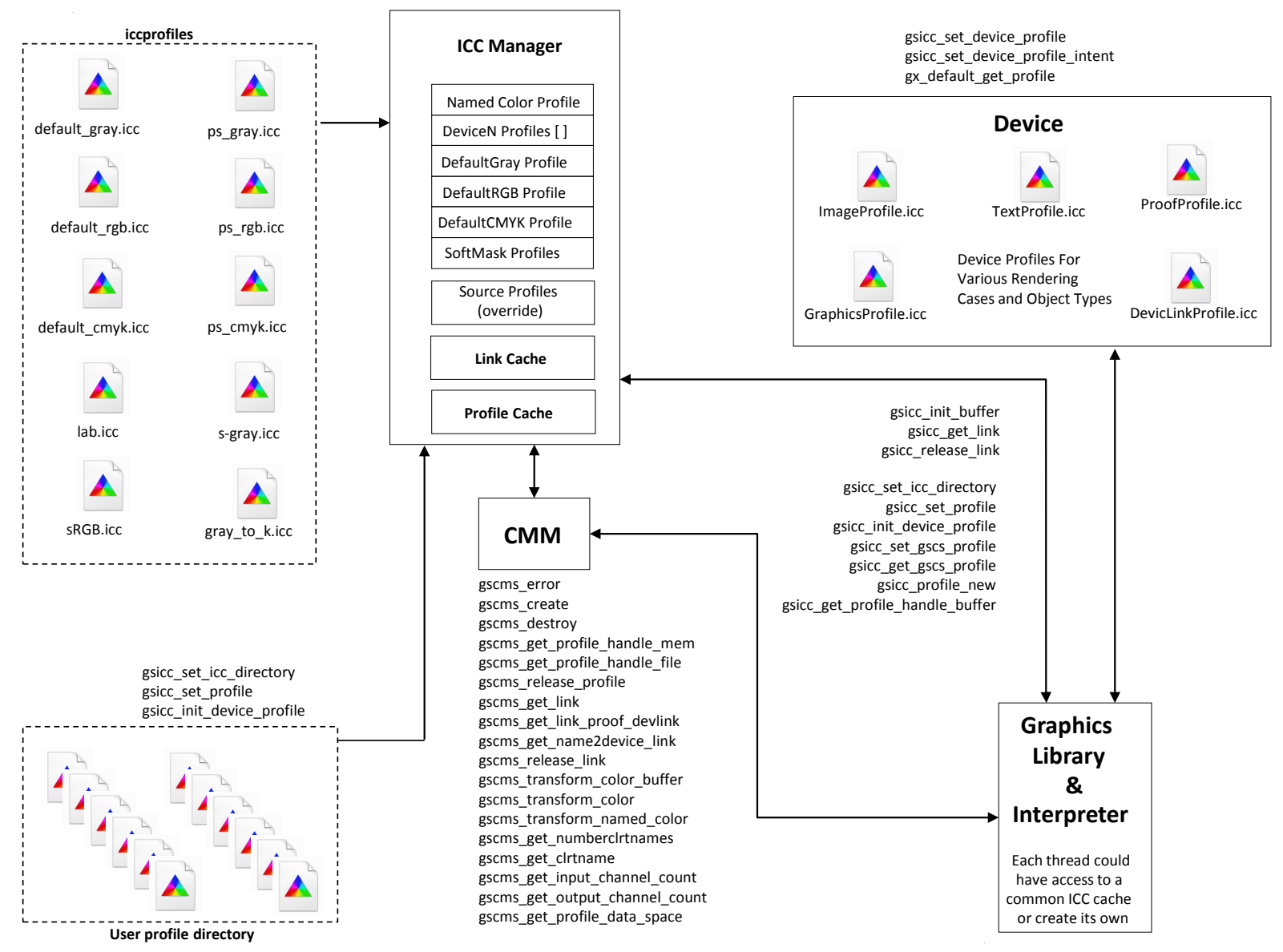

Figure 1: Graphical Overview of Ghostscript's Color Architecture 
colors (e.g. CMYK). This chart is measured using a colorimeter or a spectrophotometer. This provides the forward mapping from process colors to CIELAB values. The inverse mapping (from CIELAB to process colors) is obtained by inverting this table usually through a brute force search and extrapolation method. These mappings are both packed into an ICC format, thereby defining mappings between the device "process colors" and the CIE color space.

\section{Usage}

There are a number of command line options available for color control. These options are also available as device parameters and so can be set from Ghostscript's command prompt when Ghostscript is used in "server-mode" operation.

To define source colors that are not already colorimetrically defined in the source document, the following command line options can be invoked:

$$
\begin{aligned}
& \text {-sDefaultGrayProfile }=\text { my_gray_profile.icc } \\
& \text {-sDefaultRGBProfile }=\text { my_rgb_profile.icc } \\
& \text {-sDefaultCMYKProfile }=\text { my_cmyk_profile.icc }
\end{aligned}
$$

In this case, for example, any Device Gray source colors will be interpreted as being defined by the ICC profile my_gray_profile.icc. If these profiles are not set, default ICC profiles will be used to define undefined colors. These default profiles are contained in the directory iccprofiles and are named default_gray.icc, default_rgb.icc and default_cmyk.icc. The profile default_gray.icc is defined to provide output along the neutral axis with an sRGB linearization. The profile default_rgb.icc is the V2 sRGB ICC profile and the profile default_cmyk.icc is a SWOP CMYK ICC profile.

It is possible to have Ghostscript use the above specified ICC profiles in place of ICC profiles embedded in the document. This is achieved using

$$
\text { -dOverrideICC }=\text { true/false }
$$

which, when set to true overrides any ICC profiles contained in the source document with the profiles specified by DefaultGrayProfile, DefaultRGBProfile, DefaultCMYKProfile. Note that if no profiles are specified for the default Device color spaces, then the system default profiles will be used for DeviceGray, DeviceRGB and DeviceCMYK source colors. For detailed override control in the specification of source colors see SourceObjectICC. 
In addition to being able to define undefined source colors, it is possible to define the ICC profile for the output device using

-sOutputICCProfile = my_device_profile.icc

Care should be taken to make sure that the number of components associated with the output device is the same as the number of components for the output device ICC profile (i.e. use an RGB profile for an RGB device). If the destination device is CMYK + SPOT colorants, then it is possible to specify either a CMYK ICC profile or an N-Color ICC profile for the device. If a CMYK profile is specified, then only the CMYK colorants will be color managed. If an output profile is not specified, then the default CMYK profile is used as the output profile.

If an N-Color (NCLR) ICC profile is specified for the output device (valid for tiffsep and psdcmyk devices), then it is possible to specify the name of the colorants in the profile. This specification is done using

-sICCOutputColors= "Cyan, Magenta, Yellow, Black, Orange, Violet"

where the colorants listed are shown as an example. The list of the colorant names must be in the order that they exist in the profile. Note that if a colorant name that is specified for the profile occurs also within the document (e.g. "Orange" above), then these colorants will be associated with the same separation. It is possible through a compile time option LIMIT_TO_ICC defined in gdevdevn.h to restrict the output colorants of the psdcmyk and tiffsep device to the colorants of the ICC profile or to allow additional spot colorants in the document to be created as different separations. If restricted, the other spot colorants will go through the alternate tint transform and then be mapped to the color space defined by the N-CLR profile.

Note that if an NCLR profile is specified for the device and -sICCOutputColors is not specified, then the assumption will be that the first four colorants in the profile are cyan, magenta, yellow and black and the remaining spot colors will be named using the form ICC_COLOR $i$ where $i$ is an index from 0 to the number of spot colors in the profile minus one.

A directory can be defined, which will be searched to find the above defined ICC profiles. This makes it easier for users who have their profiles contained in a single directory and do not wish to append the full path name in the above command line options. The directory is set using

$$
\text { -sICCProfilesDir = c:/my_icc_profiles }
$$


Note that if the build of gs or other PDL languages is performed with COMPILE_INITS=1, then the profiles contained in gs/iccprofiles will be placed in the ROM file system. If a directory is specified on the command line using -sICCProfilesDir=, that directory is searched before the iccprofiles/ directory of the ROM file system is searched.

Named color support for separation color spaces is specified through the command line option

-sNamedProfile $=\mathrm{c}: /$ my_namedcolor_structure

While the ICC specification does define a named color format, the above structure can in practice be much more general for those who have complex handling requirements of separation color spaces. For example, some developers wish to use their own proprietary-based format for spot color management. This command option is for developer use when an implementation for named color management is designed for the function gsicc_transform_named_color located in gsicc_cache.c . An example implementation is currently contained in the code [see comments above gsicc_transform_named_color in gsicc_cache.c]. For the general user, this command option should really not be used.

The above option deals with the handling of single spot (Separation) colors as well as with DeviceN colors. An example of its use for DeviceN and Separation colors is given in gs/toolbin/color/named_color, where you will want to use the command line option sNamedProfile=named_color_table.txt.

It is also possible to specify ICC profiles for managing DeviceN source colors. This is done using the command line option

-sDeviceNProfile = c:/my_devicen_profile.icc

Note that neither PS nor PDF provide in-document ICC profile definitions for DeviceN color spaces. With this interface it is possible to provide this definition. The colorants tag order in the ICC profile defines the lay-down order of the inks associated with the profile. A windowsbased tool for creating these source profiles is contained in gs/toolbin/color/icc_creator. If non-ICC based color management of DeviceN source colors is desired by a developer, it is possible to use the same methods used for the handling of individual spot colors as described above.

The command line option

$$
\text { -sProofProfile }=\text { my_proof_profile.icc }
$$


enables the specification of a proofing profile, which will make the color management system link multiple profiles together to emulate the device defined by the proofing profile. See Section 4.3 for details on this option.

The command line option

$$
\text { -sDeviceLinkProfile }=\text { my_link_profile.icc }
$$

makes it possible to include a device link profile in the color transformations. This is useful for work flows where one wants to map colors first to a standard color space such as SWOP or Fogra CMYK, but it is desired to redirect this output to other CMYK devices. See Section 4.3 for details on this option.

It is possible for a document to specify the rendering intent to be used when performing a color transformation. Ghostscript is set up to handle four rendering intents with the nomenclature of Perceptual, Colorimetric, Saturation, and Absolute Colorimetric, which matches the terminology used by the ICC format. By default, per the specification, the rendering intent is Relative Colorimetric for PDF and PS documents. In many cases, it may be desired to ignore the source settings for rendering intent. This is achieved through the use of

\section{-dRenderIntent $=$ intent}

which sets the rendering intent that should be used with the profile specified above by sOutputICCProfile. The options for intent are 0, 1, 2 and 3, which correspond to the ICC intents of Perceptual, Colorimetric, Saturation, and Absolute Colorimetric.

Similarly, it is possible to turn off or on black point compensation for the color managed objects in the document. Black point compensation is a mapping performed near the black point that ensures that the luminance black in a source color space is mapped to the luminance black in a destination color space with adjustments to ensure a smooth transition for near black colors. The mapping is similar to the mapping performed at the white point between devices. With black point compensation enabled, potential loss of detail in the shadows is reduced. By default, Ghostscript has black point compensation enabled. However, note that the PDF 2.0 specification adds a black point compensation member to the extended graphic state. As such, it is possible that the document could turn off black point compensation. If this is not desired, it is possible to force black point compensation to a particular state using

$$
\text { -dBlackPtComp }=0 / 1
$$


where 0 implies compensation is off and 1 implies that compensation if on. Integer values were used instead of boolean for this command to enable easy expansion of the option to different types of black point compensation methods.

It is also possible to make use of the special black preserving controls that exist in littleCMS. The command line option

-dKPreserve $=0 / 1 / 2$

specifies if black preservation should be used when mapping from CMYK to CMYK. When using littleCMS as the CMM, the code 0 corresponds to no preservation, 1 corresponds to the PRESERVE_K_ONLY approach described in the littleCMS documentation and 2 corresponds to the PRESERVE_K_PLANE approach. See the lcms users manual for details on what these options mean.

Ghostscript currently provides overprint simulation for spot colorants when rendering to the separation devices psdcmyk and tiffsep. These devices maintain all the spot color planes and merge these together to provide a simulated preview of what would be printed. Work is currently under development to provide overprint simulation to the other devices through the use of an intermediate compositing device.

There are three additional special color handling options that may be of interest to some users. One is

\section{-dDeviceGrayToK = true/false}

By default, Ghostscript will map DeviceGray color spaces to pure K when the output device is CMYK based. The gray_to_k.icc profile in ./profiles is used to achieve this mapping of source gray to the colorant $\mathrm{K}$. The mapping of gray to $\mathrm{K}$ may not always be desired. In particular, it may be desired to map from the gray ICC profile specified by -sDefaultGrayProfile to the output device profile. To achieve this, one should specify -dDeviceGrayToK=false.

In certain cases, it may be desired to not perform ICC color management on DeviceGray, DeviceRGB and DeviceCMYK source colors. This can occur in particular if one is attempting to create an ICC profile for a target device and needed to print pure colorants. In this case, one may want instead to use the traditional Postscript 255 minus operations to convert between RGB and CMYK with black generation and undercolor removal mappings. To achieve these types of color mappings use the following command set to true

$$
\text { -dUseFastColor }=\text { true/false }
$$




\subsection{Output Intents and Post Rendering Color Management}

PDF documents can contain target ICC profiles to which the document is designed to be rendered. These are called output intents within the PDF specification. It is possible to make use of these profiles with the use of the command line option

\section{-dUsePDFX3Profile $=$ int}

If this option is included in the command line, source device color values (e.g DeviceCMYK, DeviceRGB, or DeviceGray) that match the color model of the output intent will be interpreted to be in the output intent color space. In addition, if the output device color model matches the output intent color model, then the destination ICC profile will be the output intent ICC profile. If there is a mismatch between the device color model and the output intent, the output intent profile will be used as a proofing profile, since that is the intended rendering. Note that a PDF document can have multiple rendering intents per the PDF specification. As such, with the option -dUsePDFX3Profile the first output intent encountered will be used. It is possible to specify a particular output intent where int is an integer (a value of 0 is the same as not specifying a number). Probing of the output intents for a particular file is possible using extractICCprofiles.ps in ./gs/toolbin. Finally, note that the ICC profile member entry is an option in the output intent dictionary. It is possible for the output intent dictionary to specify a registry and a standard profile (e.g. Fogra39) instead of providing a profile. Ghostscript will not make use of these output intents. Instead, if desired, these standard profiles should be used with the commands specified above (e.g. -sOutputICCProfile). Note that it is possible that a rendering intent can be an NCLR profile. In this case, it is necessary to ensure that the device can handle such a profile (e.g. the psdcmyk and tiffsep devices). In addition, the colorant names should be specified using -sICCOutputColors.

When using the output intent, but going to an output color space that is different than the actual intent, it may be desirable to apply an ICC transformation on the rendered output buffer. For example, it may be necessary to render to the output intent ICC profile color space to ensure proper color blending, overprinting and that other complex operations occur as intended by the document author. Once the document is rendered, we would want to transform to the color space defined for our actual output device. The tiffscaled devices as well as the tiffsep device allow the specification of a post render ICC profile to achieve this. The command line option is made using

-sPostRenderProfile $=$ my_postrender_profile.icc 
Note that this allows for the cases where the output intent color space of the document is CMYK based while the output device is RGB based. In such a situation we would use a PostRenderProfile that was RGB based.

\subsection{Transparency and Color Management}

Transparency blending in PDF can be dependent upon the color space in which the blending takes place. In certain source files, the color space for which the blending is to occur is not specified. Per the specification, when this occurs, the color space of the target device should be used. For consistent output across different device types this is not always desirable. For this reason, Ghostscript provides the capability to specify the desired default blending color space through the command line option

-sBlendColorProfile $=$ my_blend_profile.icc

When this option is used, if a source file has transparency blending the blending result should not depend upon the output device color model.

Ghostscript has the capability to maintain object type information even through transparency blending. This is achieved through the use of a special tag plane during the blending of the objects. When the final blending of the objects occurs this tag information is available. Mixed objects will be indicated as such (e.g text blended with image). A device can have a specialized put_image operation that can handle the pixel level color management operation and apply the desired color mapping for different blend cases. The bittagrgb device in Ghostscript provides a demonstration of the use of the tag information.

\subsection{Proof and Device-Link Profiles}

As shown in Figure 1, the proofing profile and the device link profile are associated with the device. If these profiles have been specified using the options -sProofProfile $=$ my_proof_profile.icc and - sDeviceLinkProfile $=$ my_link_profile.icc, then when the graphics library maps a source color defined by the ICC profile source.icc to the device color values, a transformation is computed by the CMM that consists of the steps shown in Figure 2. In this Figure, Device ICC Profile is the ICC profile specified for the actual device (this can be specified using -sOutputICCProfile). In practice, the CMM will create a single mapping that performs the transformation of the multiple mappings shown in Figure 2. If we specify a proofing profile, then our output should provide a proof of how the output would appear if it had been displayed or printed on the proofing device defined by the proofing profile. The device link profile is useful for cases where one may have a work flow that consists of always rendering to a common CMYK space such as Fogra 39 followed by a mapping with a specialized device 
link profile. In this case, the profile specified by -sOutputICCProfile would be the profile for the common CMYK space.

Note that if -sSourceObjectICC is used to specify device link ICC profiles to map from source color spaces to device colors, then it is not possible to use either the device profile or the proofing profile for these objects. However, a device link profile that is associated with the target device will be merged with the device link profile specified for the source object.

\subsection{Object dependent color management}

It is often desired to perform unique mappings based upon object types. For example, one may want to perform one color transformation on text colors to ensure a black text and a different transformation on image colors to ensure perceptually pleasing images and yet another transformation on graphics to create saturated colors. To achieve this, Ghostscript provides an unprecedented amount of color control based upon object type.

The following commands, enable one to specify unique output ICC profiles, rendering intents, black point compensation and black preserving methods for text, graphic and image objects. As shown in Figure 1, these profiles are stored in the device structure. Specifically, the command options are:

\section{-sGraphicICCProfile = filename}

Sets the ICC profile that will be associated with the output device for vector-based graphics (e.g. solid color Fill, Stroke operations). This option can be used to obtain more saturated colors for graphics. Care should be taken to ensure that the number of colorants associated with the device is the same as the profile.

-dGraphicIntent $=$ intent

Sets the rendering intent that should be used with the profile specified above by -sGraphicICCProfile. The options are the same as specified for -dRenderIntent.

$$
\text { -dGraphBlackPt }=0 / 1
$$

Sets the black point compensation setting that should be used with the profile specified above by -sGraphicICCProfile.

$$
\text { -dGraphicKPreserve }=0 / 1 / 2
$$

specifies the black preserving method that should be used from mapping CMYK to CMYK for graphic objects. The options are the same as specified for -dKPreserve. 


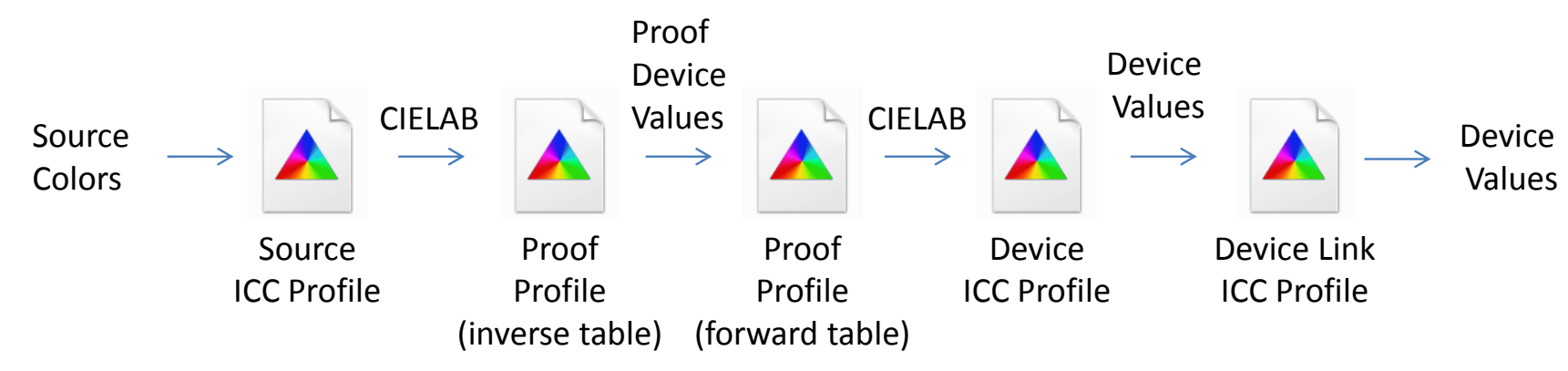

Figure 2: Flow of data through source, proof, destination and device link ICC profiles 


$$
\text { -sImageICCProfile }=\text { filename }
$$

Sets the ICC profile that will be associated with the output device for images. This can be used to obtain perceptually pleasing images. Care should be taken to ensure that the number of colorants associated with the device is the same as the profile.

$$
\text { -dImageIntent }=\text { intent }
$$

Sets the rendering intent that should be used with the profile specified above by -sImageICCProfile. The options are the same as specified for -dRenderIntent.

$$
\text { -dImageBlackPt }=0 / 1
$$

Sets the black point compensation setting that should be used with the profile specified above by -sImageICCProfile.

$$
\text { -dImageKPreserve }=0 / 1 / 2
$$

specifies the black preserving method that should be used from mapping CMYK to CMYK for image objects. The options are the same as specified for -dKPreserve.

$$
- \text { sTextICCProfile }=\text { filename }
$$

Sets the ICC profile that will be associated with the output device for text. This can be used ensure K only text at the output. Care should be taken to ensure that the number of colorants associated with the device is the same as the profile.

$$
\text { -dTextIntent }=\text { intent }
$$

Sets the rendering intent that should be used with the profile specified above by -sTextICCProfile. The options are the same as specified for -dRenderIntent.

$$
-\mathrm{dTextBlackPt}=0 / 1
$$

Sets the black point compensation setting that should be used with the profile specified above by -sTextICCProfile.

$$
\text { -dTextKPreserve }=0 / 1 / 2
$$


specifies the black preserving method that should be used from mapping CMYK to CMYK for text objects. The options are the same as specified for -dKPreserve.

In addition to being able to have the output ICC profile dependent upon object type, it is possible to have the source ICC profile and rendering intents be dependent upon object types for GRAY, RGB and CMYK objects. Because this requires the specification of many new parameters and is only used in specialized situations, the specification is made through a single text file. The text file is specified to Ghostscript using

\section{-sSourceObjectICC = filename}

This option provides an extreme level of override control to specify the source color spaces, rendering intents and black point compensation to use with graphics, images and text for source objects. The specification is made through a file that contains on a line, a key name to specify the object type (e.g. Image_CMYK) followed by an ICC profile file name, a rendering intent number ( 0 for perceptual, 1 for colorimetric, 2 for saturation, 3 for absolute colorimetric), a black point compensation value (0 or 1), a boolean to indicate if source ICC profiles should be overridden, and a value for the CMYK objects to indicate if any type of black preservation should be used when going from CMYK to CMYK transformations. An example file is given in ./gs/toolbin/color/src_color/objsrc_profiles_example.txt. Profiles to demonstrate this method of specification are also included in this folder. Note that if objects are colorimetrically specified through this mechanism, other operations like -sImageIntent, -dOverrideICC, have no affect.

The example file mentioned above contains the following tab delimited lines

$\begin{array}{llllll}\text { Graphic_CMYK } & \text { cmyk_src_cyan.icc } & 0 & 1 & 0 & 0 \\ \text { Image_CMYK } & \text { cmyk_src_magenta.icc } & 0 & 1 & 0 & 0 \\ \text { Text_CMYK } & \text { cmyk_src_yellow.icc } & 0 & 1 & 0 & 0 \\ \text { Graphic_RGB } & \text { rgb_source_red.icc } & 0 & 1 & 0 & \\ \text { Image_RGB } & \text { rgb_source_green.icc } & 0 & 1 & 0 & \\ \text { Text_RGB } & \text { rgb_source_blue.icc } & 0 & 1 & 0 & \end{array}$

where the first item in the line is the key word, the second item in the line is the file name of the source ICC profile to use for that object type, the third item specifies the rendering intent, the fourth item specifies the black point compensation setting, the fifth term indicates if source ICC profiles should be overridden, and the sixth term which should only be there for CMYK source objects indicates if any type of black preservation should be performed if we are going to a CMYK color space. Note that not all types need to be specified. It is possible to have only a single type specified in the file (e.g. Image_CMYK). The other items would 
render in a normal default fashion in this case. Note that it is necessary to include all the possible options in each line. That is, "Graphic_CMYK cmyk_src_cyan.icc 0" is not a valid line but must include settings for the next three values as given above for Graphic_CMYK. In addition to CMYK and RGB types given above, the user can also specify Graphic_GRAY, Image_GRAY and Text_GRAY objects.

In addition, it is possible to have unique color management methods for these object types through two special names which are "None" and "Replace". For example, if our file contained the following two lines

\section{Graphic_CMYK None \\ Text_CMYK Replace}

then graphic CMYK source objects will not be color managed but instead will go through the standard Postscript mapping methods (e.g. 255-X). CMYK text objects will go through the color replacement color management route which is provided for those developers who wish to provide direct output replacement colors for a given incoming source color. This is currently implemented in the function gsicc_rcm_transform_general, which is in the file gsicc_replacecm.c. The current implementation computes the color negative of the source color as a demonstration. Note that the replaced color should be in the device's color space. The entire contents of the file, gsicc_replacecm.c are provided as an example for developers.

In addition, one can specify a device link ICC profile to use with a particular source object type when mapping to the destination color space. This is done by simply using a notation such as

\section{Graphic_RGB linkRGBtoCMYK.icc $\quad 0 \quad 1 \quad 0$}

in the -sSourceObjectICC file, where linkRGBtoCMYK.icc is the device link ICC profile file name. Note that the rendering intent etc are still specified but their effect is dependent upon the CMM that is hooked in with Ghostscript. With the current use of lcms, these values have no effect with device link profiles. Note also that if the device ICC profile is an NCLR profile, it is possible that the device link profiles specified in the -sSourceObjectICC file can have a destination color space that is either CMYK or NCLR.

For those interested in this level of control, it is recommended to execute a number of examples. In the first example, copy the files in ./gs/toolbin/color/src_color/ to ./iccprofiles and render the file ./examples/text_graph_image_cmyk_rgb.pdf with the option sSourceObjectICC = objsrc_profiles_example.txt to an RGB device (e.g. tiff24nc). Note, to ensure that Ghostscript can find all the files and to avoid having to do a full rebuild to create the ROM file system, you may want to specify the icc directory using

-sICCProfilesDir="your_full_path_to_iccprofiles/", which provides the full path to ./iccprofiles/. Windows users should be sure to use the forward slash delimiter due to the special 


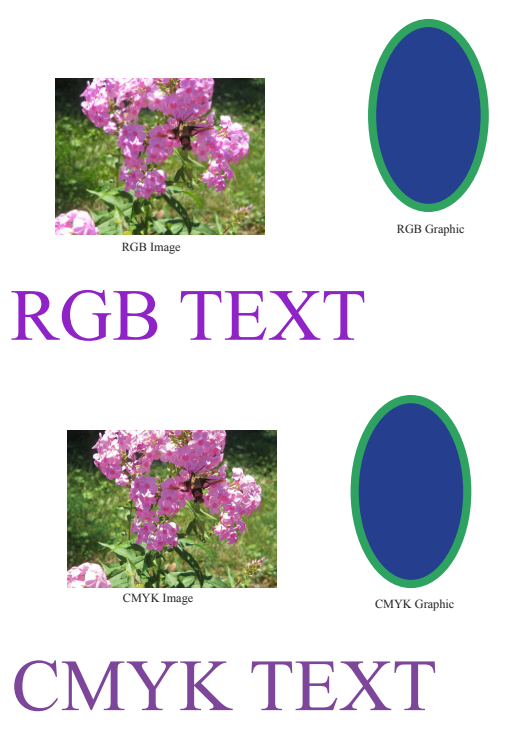

Figure 3: Example file with mixed content. The file includes RGB and CMYK text, graphics, and iamges

interpretation of "" by the Microsoft C startup code.

Figure 3 displays the source file text_graph_image_cmyk_rgb.pdf rendered with default settings and Figure $4 \mathrm{a}$ displays the result when rendered using -sSourceObjectICC $=$ objsrc_profiles_example.txt. The profiles specified in objsrc_profiles_example.txt are designed to render object types to the color specified in their name when used as a source profile. In this case, RGB graphics, images and text are rendered red, green and blue respectively and CMYK graphics, images and text are rendered cyan, magenta and yellow respectively.

Modifying the contents of the objsrc_profiles_example.txt file to

$\begin{array}{llllll}\text { Graphic_CMYK } & \text { cmyk_src_renderintent.icc } & 0 & 1 & 0 & 0 \\ \text { Image_CMYK } & \text { cmyk_src_renderintent.icc } & 1 & 1 & 0 & 0 \\ \text { Text_CMYK } & \text { cmyk_src_renderintent.icc } & 2 & 1 & 0 & 0\end{array}$

and rendering the file./examples/text_graph_image_cmyk_rgb.pdf to an RGB device, one obtains the output shown in Figure $4 \mathrm{~b}$. In this case, we demonstrated the control of rendering intent based upon object type. The profile cmyk_src_renderintent.icc is designed to create significantly different colors for its different intents. Since we only specified this for the CMYK objects we see that they are the only objects effected and that this profile renders 


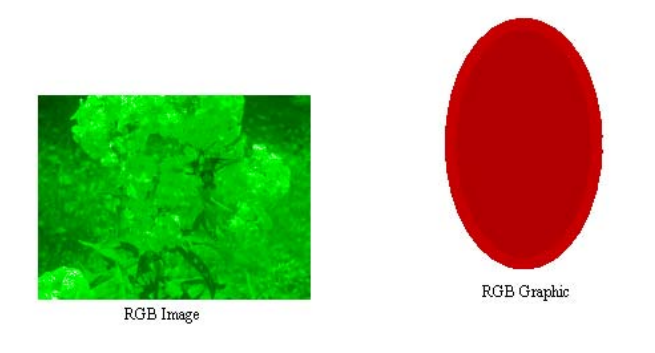

\section{RGB TEXT}

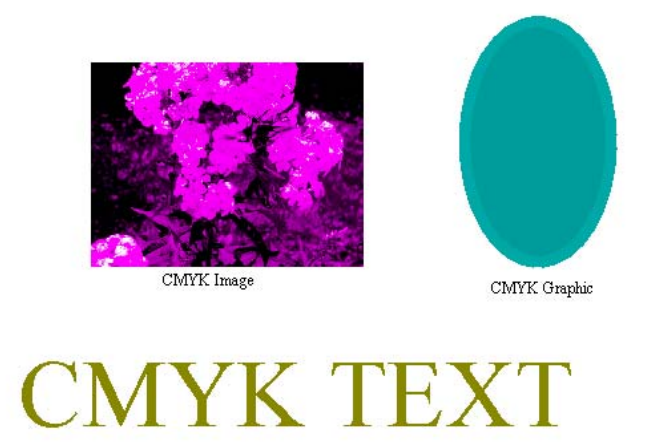

(a) Source profiles vary with object type
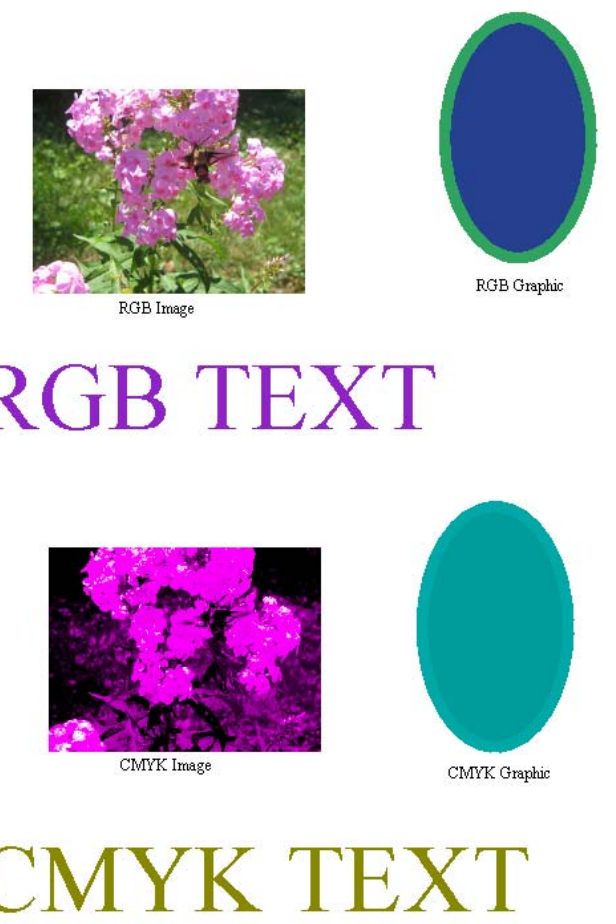

(b) Rendering intents vary with CMYK source object type

Figure 4: Examples of object based color transformations for the file from Figure 3 by specifying source profiles and/or rendering intents 

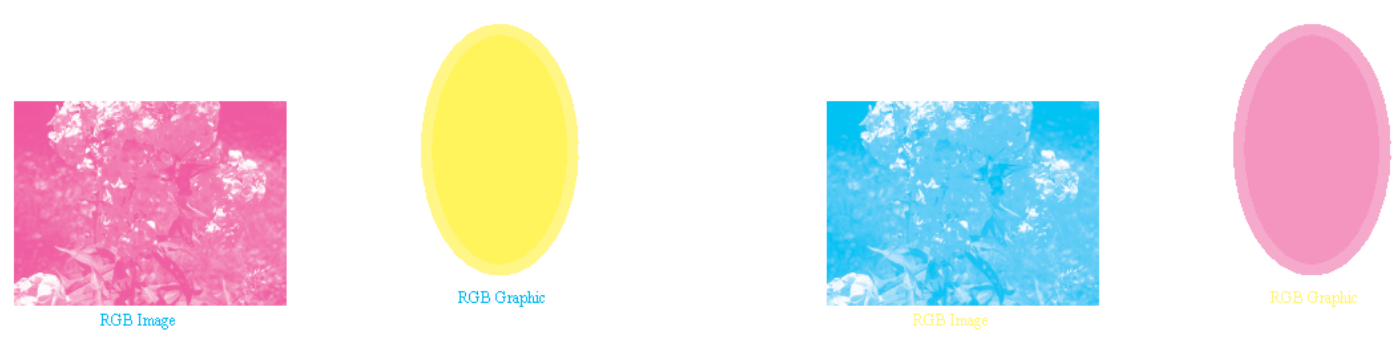

\section{RGB TEXT}

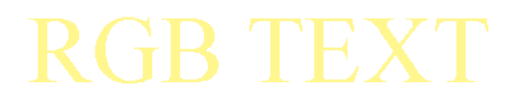

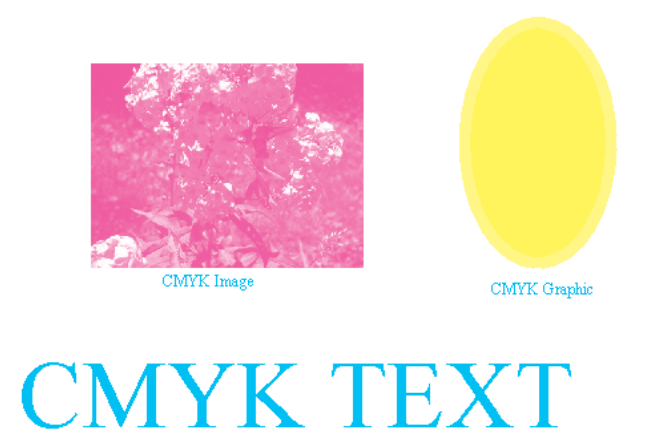

(a) Destination profiles vary with object type
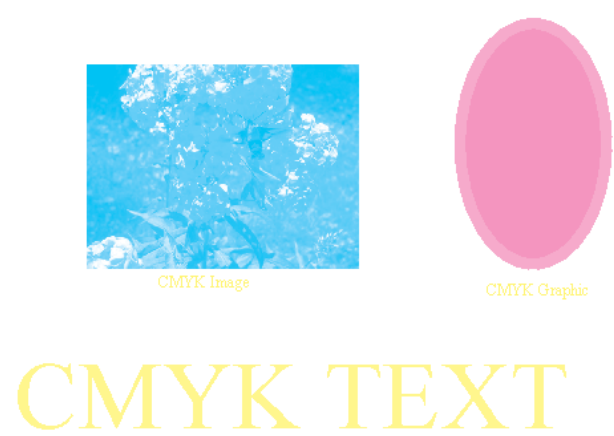

Figure 5: Examples of object based color transformations for the file from Figure 3 by specifying destination profiles and/or intents 


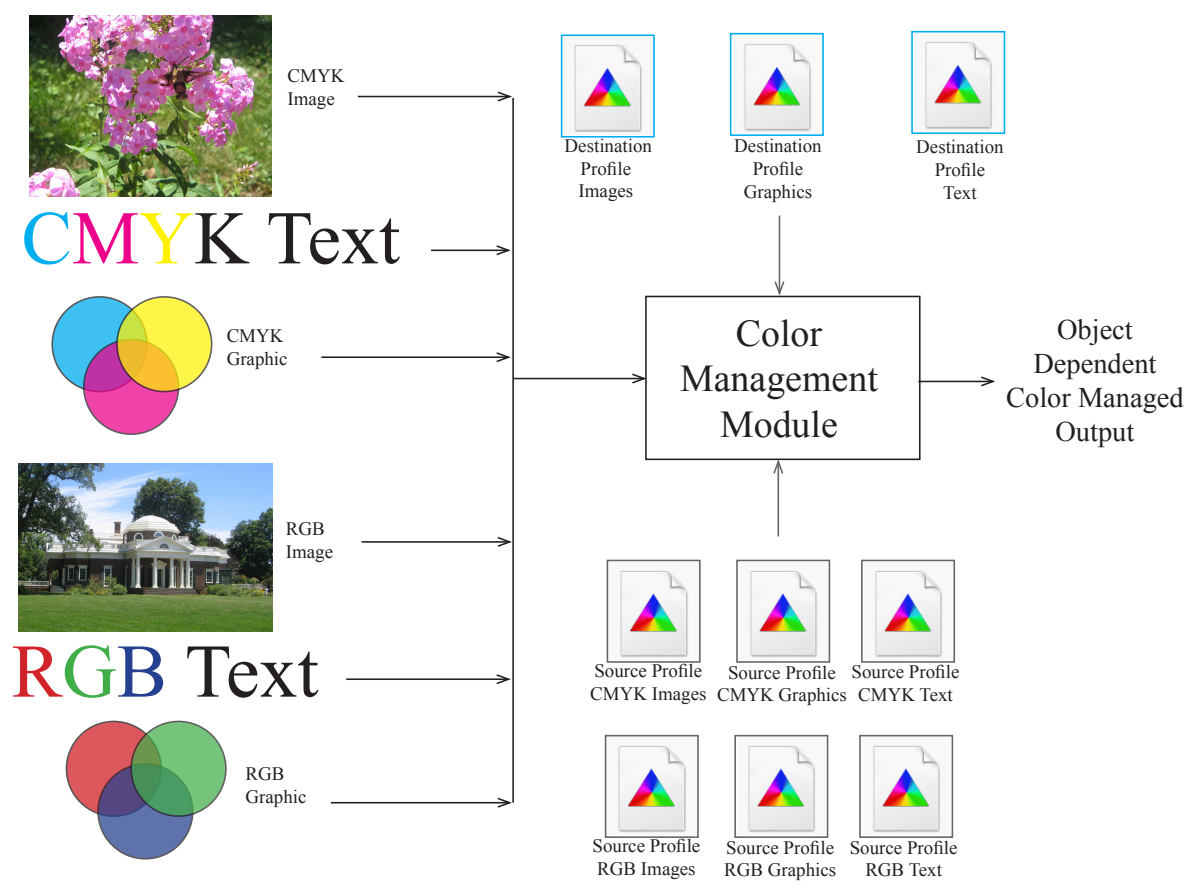

Figure 6: Overview of profiles that can be used in object dependent color management

its perceptual intent cyan, its colorimetric intent magenta and its saturation intent yellow.

For another example of object dependent color management, copy the files in ./toolbin/color/icc_creator/effects to ./iccprofiles. Now specify unique output ICC profiles for different object types using the command line options

-sGraphicICCProfile $=$ yellow_output.icc

-sImageICCProfile = magenta_output.icc

-sTextICCProfile = cyan_output.icc

while rendering the file text_graph_image_cmyk_rgb.pdf to a CMYK device (e.g. tiff32nc). Figure 5a displays the results. In this case, the profiles, cyan_output.icc, yellow_output.icc and magenta_output.icc render a color that is indicated by their name when used as an output profile.

Finally, in yet another example, we can demonstrate the effect of rendering intent for different objects using the command line options 


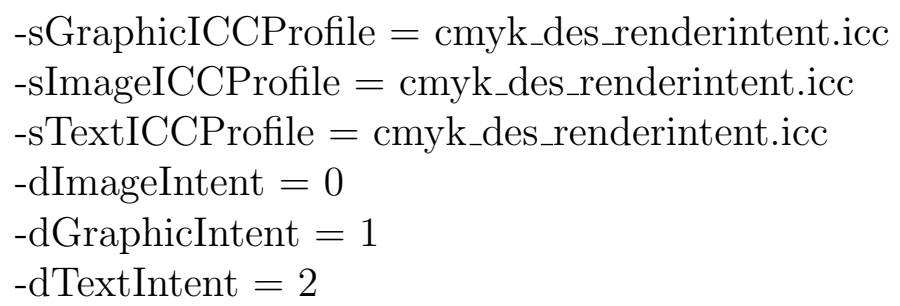

Figure 5b displays the result. The profile cmyk_des_renderintent.icc is designed such that the perceptual rendering intent outputs cyan only, the colorimetric intent outputs magenta only and the saturation intent outputs yellow only.

A graphical overview of the object dependent color control is shown in Figure 6, which shows how both the source and/or the destination ICC profiles can be specified.

\section{Details of objects and methods}

At this point, let us go into further detail of the architecture and in particular the various functions that may be of interest to those wishing to work with ICC profiles within Ghostscript. Following this, we will discuss the requirements for interfacing another CMM to Ghostscript as well as details for customization of handling Separation and DeviceN color spaces.

\subsection{ICC Manager}

The ICC Manager is a reference counted member variable of Ghostscript's imager state. Its functions are to:

- Store the required profile information to use for Gray, RGB, and CMYK source colors that are NOT colorimetrically defined in the source document. These entries must always be set in the manager and are set to default values unless defined by the command line interface.

- Store the optional profile/structure information related to named colors and DeviceN colors.

- Store the CIELAB source profile.

- Store the specialized profile for mapping gray source colors to K-only CMYK values.

- Store settings for profile override, output rendering intent (i.e. perceptual, colorimetric, saturation or absolute colorimetric) and source color rendering intents. 
- Store the profiles that are used for softmask rendering if soft masks are contained in the document.

- Store the profiles used for object dependent source color specification through the use of -sSourceObjectICC.

- Store the boolean flags for profile and rendering intent override of source settings.

The manager is created when the imaging state object is created for the graphics library. It is reference counted and allocated in garbage collected (GC) memory that is stable with graphic state restores. The default GRAY, RGB and CMYK ICC color spaces are defined immediately during the initialization of the graphics library. If no ICC profiles are specified externally, then the ICC profiles that are contained in the root folder iccprofiles will be used. The ICC Manager is defined by the structure given below.

typedef struct gsicc_manager_s \{ cmm_profile_t $*$ device_named; cmm_profile_t *default_gray; cmm_profile_t $*$ default_rgb; cmm_profile_t *default_cmyk; cmm_profile_t *lab_profile; cmm_profile_t $*$ xyz_profile; cmm_profile_t $*$ graytok_profile; gsicc_devicen_t $*$ device_n; gsicc_smask_t ${ }^{*}$ smask_profiles; bool override_internal;

/* The named color profile for the device $* /$

/* Default gray profile for device gray */

/* Default RGB profile for device RGB */

/* Default CMYK profile for device CMKY * /

$/ *$ Colorspace type ICC profile from LAB to LAB */

/* RGB based profile that hands back CIEXYZ values */

/* A specialized profile for mapping gray to $\mathrm{K} *$ /

/* A linked list of profiles used for DeviceN support */

/* Profiles used when we are in a softmask group */

$/ *$ Set via the user params */

cmm_srcgtag_profile_t *srcgtag_profile; /* Object dependent source profiles */

rc_header rc;

\} gsicc_manager_t;

Operators that relate to the ICC Manager are contained in the file gsicc_manage.c/h and include the following:

gsicc_manager_t* gsicc_manager_new(gs_memory_t *memory);

Creator for the ICC Manager. 
int gsicc_init_iccmanager(gs_state ${ }^{*}$ pgs);

Initializes the ICC Manager with all the required default profiles.

int gsicc_set_profile(gsicc_manager_t *icc_manager, const char *pname, int namelen, gsicc_profile_t defaulttype);

This is used to set the default related member variables in the ICC Manager. The member variable to set is specified by defaulttype.

cmm_profile_t* gsicc_finddevicen(const gs_color_space *pcs, gsicc_manager_t *icc_manager);

Search the DeviceN profile array contained in the ICC Manager for a profile that has the same colorants as the DeviceN color space in the PDF or PS document.

Several ICC profile-specific operators in gsicc_manage.c/h that may be of interest to developers include the following:

cmm_profile_t* gsicc_profile_new(stream *s, gs_memory_t *memory, const char* pname, int namelen);

Returns an ICC object given a stream pointer to the ICC content. The variables pname and namelen provide the filename and name length of the stream if it is to be created from a file. If the data is from the source stream, pname should be NULL and namelen should be zero.

int gsicc_clone_profile(cmm_profile_t * ${ }_{\text {source, }}$ cmm_profile_t ${ }^{* *}$ destination, gs_memory_t *memory);

Used for cloning an ICC profile. This is used in the multi-threaded rendering case to create thread-safe color management as the threads render to the same device profile. 
void gsicc_init_hash_cs $\left(\mathrm{cmm}_{\text {_p }}\right.$ grofile_t ${ }^{*}$ picc_profile, gs_imager_state ${ }^{*}$ pis $)$;

Set the hash code for a profile.

int64_t gsicc_get_hash(cmm_profile_t * profile);

Get the hash code for a profile. In gsicc_cache.h/c due to its use in computing links.

gcmmhprofile_t gsicc_get_profile_handle_clist(cmm_profile_t *picc_profile, gs_memory_t *memory);

For a profile that is embedded inside the c-list, obtain a handle from the CMM.

gcmmhprofile_t gsicc_get_profile_handle_buffer(unsigned char *buffer, int profile_size);

For a profile that is contained in a memory buffer, obtain a handle from the CMM.

cmm_profile_t* gsicc_get_profile_handle_file(const char* pname, int namelen, gs_memory_t *mem);

Given a profile file name, obtain a handle from the CMM.

void gsicc_init_profile_info(cmm_profile_t ${ }^{*}$ profile);

With a profile handle already obtained from the CMM, set up some of the member variables in the structure cmm_profile_t.

void gsicc_profile_serialize(gsicc_serialized_profile_t * profile_data, cmm_profile_t $*$ iccprofile); 
A function used to serialize the icc profile information for embedding into the c-list (display list).

cmm_profile_t* gsicc_read_serial_icc(gx_device ${ }^{*}$ dev, int64_t icc_hashcode);

Read out the serialized icc data contained in the c-list for a given hash code.

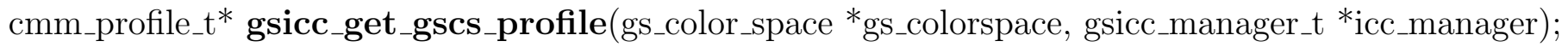

Returns the cmm_icc_profile_data member variable of the gs_color_space object.

int gsicc_set_gscs_profile(gs_color_space $*$ pcs, cmm_profile_t $*_{\text {icc_profile, }}$ gs_memory_t $*$ mem);

Sets the member variable cmm_icc_profile_data of the gs_color_space object (pointed to by pcs) to icc_profile.

unsigned int gsicc_getprofilesize(unsigned char *buffer);

Get the size of a profile, as given by the profile information.

int gsicc_getsrc_channel_count(cmm_profile_t $\left.* i c c \_p r o f i l e\right)$;

Returns the number of device channels for a profile.

gs_color_space_index gsicc_get_default_type(cmm_profile_t * profile_data);

Detect profiles that were set as part of the default settings. These are needed to differentiate between embedded document ICC profiles and ones that were supplied to undefined device source colors (e.g. DeviceRGB). During high level device writing (e.g. pdfwrite), these default profiles are usually NOT written out. 
void gsicc_get_srcprofile(gsicc_colorbuffer_t data_cs, gs_graphics_type_tag_t graphics_type_tag, cmm_srcgtag_profile_t ${ }^{*}$ srcgtag_profile, cmm_profile_t $* *$ profile, gsicc_rendering_intents_t *rendering_intent);

Given a particular object type this function will return the source profile and rendering intent that should be used if it has been specified using -sSourceObjectICC.

\subsection{Device Profile Structure}

The device structure contains a member variable called icc_struct, which is of type ${ }^{*} \mathrm{cmm} \_$dev_profile_t. The details of this structure are shown below.

typedef struct cmm_dev_profile_s \{ cmm_profile_t $*$ device_profile[] /* Object dependent (and default) device profiles */ cmm_profile_t * proof_profile; cmm_profile_t $*$ link_profile;

/* The proof profile $* /$

/* The device link profile $* /$

cmm_profile_t $*$ oi_profile; cmm_profile_t $*$ blend_profile; cmm_profile_t * postren_profile;

/* Output intent profile */

/* blending color space */

/* Profile for use by devices post render */ gsicc_rendering_param_t rendercond[]; bool devicegraytok;

bool graydetection; bool pageneutralcolor; bool usefastcolor; bool supports_devn; bool sim_overprint; gsicc_namelist_t * spotnames; bool prebandthreshold; gs_memory_t *memory; rc_header rc;

\} cmm_dev_profile_t;

There are a number of operators of interest associated with the device profiles that may be of use for developers. These include:

cmm_dev_profile_t* ${ }^{*}$ gsicc_new_device_profile_array(gs_memory_t ${ }^{*}$ memory); 
This allocates the above structure.

int gsicc_set_device_profile(gx_device ${ }^{*}$ pdev, gs_memory_t ${ }^{*}$ mem, char ${ }^{*}$ file_name, gsicc_profile_types_t defaulttype);

This sets a device profile for a particular object type, default type, output intent, post-render, blending color space, proofing or link. This is used by gsicc_init_device_profile_struct, which will specify the default profile to this function if one was not specified.

int gsicc_init_device_profile_struct(gx_device ${ }^{*}$ dev, char * profile_name, gsicc_profile_types_t profile_type);

This sets the device profiles. If the device does not have a defined profile, then a default one is selected.

int gsicc_set_device_profile_intent(gx_device *dev, gsicc_profile_types_t intent, gsicc_profile_types_t profile_type);

This sets the rendering intent for a particular object type.

int gsicc_set_device_blackptcomp(gx_device *dev, gsicc_blackptcomp_t blackptcomp, gsicc_profile_types_t profile_type);

This sets the black point compensation for a particular object type.

int gsicc_set_device_blackpreserve(gx_device *dev, gsicc_blackpreserve_t blackpreserve, gsicc_profile_types_t profile_type);

This sets the black preservation for a particular object type. 
void gsicc_extract_profile(gs_graphics_type_tag_t graphics_type_tag, cmm_dev_profile_t * profile_struct, cmm_profile_t $* *$ profile, gsicc_rendering_param_t *render_cond);

Given a particular object type, this will return the device ICC profile and rendering conditions to use.

int gsicc_get_device_profile_comps(cmm_dev_profile_t $*$ dev_profile);

Returns the number of device components of the profile associated with the device. (Defined in gsicc_cache.h/c)

\subsection{Link Cache}

The Link Cache is a reference counted member variable of Ghostscript's imager state and maintains recently used links that were provided by the CMM. These links are handles or context pointers provided by the CMM and are opaque to Ghostscript. As mentioned above, the link is related to the rendering intents, the object type and the source and destination ICC profile. From these items, a hash code is computed. This hash code is then used to check if the link is already present in the Link Cache. A reference count variable is included in the table entry so that it is possible to determine if any entries can be removed, if there is insufficient space in the Link Cache for a new link. The Link Cache is allocated in stable GC memory and is designed with semaphore calls to allow multi-threaded c-list (display list) rendering to share a common cache. Sharing does require that the CMM be thread safe. Operators that relate to the Link Cache are contained in the file gsicc_cache.c/h and include the following:

gsicc_link_cache_t* ${ }^{*}$ sicc_cache_new $\left(g s \_m e m o r y \_t *\right.$ memory $)$;

Creator for the Link Cache. 
void gsicc_init_buffer(gsicc_bufferdesc_t *buffer_desc, unsigned char num_chan, unsigned char bytes_per_chan, bool has_alpha, bool alpha_first, bool is_planar, int plane_stride, int row_stride, int num_rows, int pixels_per_row);

This is used to initialize a gsicc_bufferdesc_t object. Two of these objects are used to describe the format of the source and destination buffers when transforming a buffer of color values.

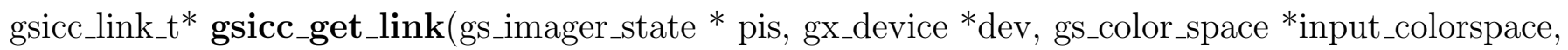
gs_color_space $*_{\text {output_colorspace, }}$ gsicc_rendering_param_t *rendering_params gs_memory_t *memory);

This returns the link given the input color space, the output color space, and the rendering intent. When the requester of the link is finished using the link, it should release the link. When a link request is made, the Link Cache will use the parameters to compute a hash code. This hash code is used to determine if there is already a link transform that meets the needs of the request. If there is not a link present, the Link Cache will obtain a new one from the CMM (assuming there is sufficient memory), updating the cache.

The linked hash code is a unique code that identifies the link for an input color space, an object type, a rendering intent and an output color space.

Note, that the output color space can be different than the device space. This occurs for example, when we have a transparency blending color space that is different than the device color space. If the output_colorspace variable is NULL, then the ICC profile associated with dev will be used as the destination color space.

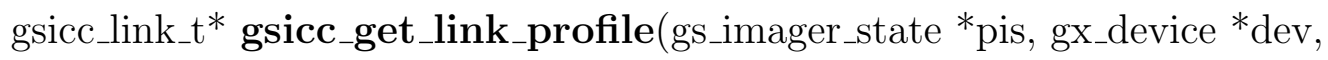
cmm_profile_t $*_{\text {gs_input_profile, }}$ cmm_profile_t $*_{\text {gs_output_profile, }}$ gsicc_rendering_param_t *rendering_params, gs_memory_t *memory, bool devicegraytok); 
This is similar to the above operation gsicc_get_link but will obtain the link with profiles that are not member variables of the gs_color_space object.

int gsicc_transform_named_color(float tint_value, byte ${ }^{*}$ color_name, uint name_size, gx_color_value device_values[], const gs_imager_state ${ }^{*}$ pis, gx_device ${ }^{*}$ dev, cmm_profile_t ${ }^{*}$ gs_output_profile, gsicc_rendering_param_t *rendering_params);

This performs a transformation on the named color given a particular tint value and returns device_values.

void gsicc_release_link $($ gsicc_link_t $*$ icclink $)$;

This is called to notify the cache that the requester for the link no longer needs it. The link is reference counted, so that the cache knows when it is able to destroy the link. The link is released through a call to the CMM.

There are special link allocation/free operations that can be invoked that are not tied to the Link Cache. These are typically used in situations where a device may need to create a link for special post rendering color management. The operations are:

gsicc_link_t* gsicc_alloc_link_dev(gs_memory_t * memory, cmm_profile_t * src_profile, cmm_profile_t $*$ des_profile, gsicc_rendering_param_t $*$ rendering_params);

This is a special allocation for a link that is used by devices for doing color management on post rendered data. It is not tied into the profile cache like gsicc_alloc_link.

void gsicc_free_link_dev(gs_memory_t *memory, gsicc_link_t* *link);

Free link allocated using gsicc_alloc_link_dev. 


\subsection{Interface of Ghostscript to CMM}

Ghostscript interfaces to the CMM through a single file. The file gsicc_littlecms2.c/h is a reference interface between littleCMS and Ghostscript. If a new library is used (for example, if littleCMS is replaced with a different CMM), the interface of these functions will remain the same, but internally they will need to be changed. Specifically, the functions are as follows:

void gscms_create(void ${ }^{* *}$ contextptr);

This operation performs any initializations required for the CMM.

void gscms_destroy(void ${ }^{* *}$ contextptr);

This operation performs any cleanup required for the CMM.

gcmmhprofile_t gscms_get_profile_handle_mem(unsigned char *buffer, unsigned int input_size);

This returns a profile handle for the profile contained in the specified buffer.

void gscms_release_profile(void *profile);

When a color space is removed or we are ending, this is used to have the CMM release a profile handle it has created.

int gscms_get_input_channel_count(gcmmhprofile_t profile); 
Provides the number of colorants associated with the ICC profile. Note that if this is a device link profile this is the number of input channels for the profile.

int gscms_get_output_channel_count(gcmmhprofile_t profile);

If this is a device link profile, then the function returns the number of output channels for the profile. If it is a profile with a PCS, then the function should return a value of three.

gcmmhlink_t gscms_get_link(gcmmhprofile_t lcms_srchandle, gcmmhprofile_t lcms_deshandle, gsicc_rendering_param_t *rendering_params);

This is the function that obtains the linkhandle from the CMM. The call gscms_get_link is usually called from the Link Cache. In the graphics library, calls are made to obtain links using gsicc_get_link, since the link may already be available. However, it is possible to use gscms_get_link to obtain linked transforms outside the graphics library. For example, this is the case with the XPS interpreter, where minor color management needs to occur to properly handle gradient stops.

gcmmhlink_t gscms_get_link_proof_devlink(gcmmhprofile_t lcms_srchandle, gcmmhprofile_t lcms_proofhandle, gcmmhprofile_t lcms_deshandle, gcmmhprofile_t lcms_devlinkhandle, gsicc_rendering_param_t *rendering_params);

This function is similar to the above function but includes a proofing ICC profile and/or a device link ICC profile in the calculation of the link transform. See Section 4.3 .

void gscms_release_link(gsicc_link_t $*$ icclink $)$;

When a link is removed from the cache or we are ending, this is used to have the CMM release the link handles it has created. 
void gscms_transform_color_buffer(gx_device ${ }^{*}$ dev, gsicc_link_t *icclink, gsicc_bufferdesc_t $*$ input_buff_desc, gsicc_bufferdesc_t ${ }^{*}$ output_buff_desc, void *inputbuffer, void * outputbuffer);

This is the function through which all color transformations on chunks of data will occur. Note that if the source hash code and the destination hash code are the same, the transformation will not occur as the source and destination color spaces are identical. This feature can be used to enable "device colors" to pass unmolested through the color processing. Note that a pointer to this function is stored in a member variable of Ghostscript's ICC link structure (gsicc_link_t.procs.map_buffer).

void gscms_transform_color(gx_device *dev, gsicc_link_t *icclink, void *inputcolor, void *outputcolor, int num_bytes);

This is a special case where we desire to transform a single color. While it would be possible to use gscms_transform_color_buffer for this operation, single color transformations are frequently required and it is possible that the CMM may have special optimized code for this operation. Note that a pointer to this function is stored in a member variable of Ghostscript's ICC link structure (gsicc_link_t.procs.map_color).

int gscms_transform_named_color(gsicc_link_t *icclink, float tint_value, const char* ColorName, gx_color_value device_values[] );

This function obtains a device value for the named color. While there exist named color ICC profiles and littleCMS supports them, the code in gsicc_littlecms.c is not designed to use that format. The named color object need not be an ICC named color profile but can be a proprietary type table. This is discussed further where -sNamedProfile is defined in the Usage section.

void gscms_get_name2device_link(gsicc_link_t *icclink, gcmmhprofile_t lcms_srchandle, gcmmhprofile_t lcms_deshandle, gcmmhprofile_t lcms_proofhandle, gsicc_rendering_param_t $*$ rendering_params,

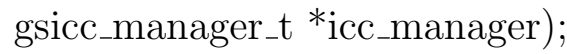


This is the companion operator to gscms_transform_named_color in that it provides the link transform that should be used when transforming named colors when named color ICC profiles are used for named color management. Since gscms_transform_named_color currently is set up to use a non-ICC table format, this function is not used.

gcmmhprofile_t gscms_get_profile_handle_file(const char *filename);

Obtain a profile handle given a file name.

char* gscms_get_clrtname(gcmmhprofile_t profile, int k);

Obtain the $k$ th colorant name in a profile. Used for DeviceN color management with ICC profiles.

int gscms_get_numberclrtnames(gcmmhprofile_t profile);

Return the number of colorant names that are contained within the profile. Used for DeviceN color management with ICC profiles.

gsicc_colorbuffer_t gscms_get_profile_data_space(gcmmhprofile_t profile);

Get the color space type associated with the profile.

int gscms_get_channel_count(gcmmhprofile_t profile);

Return the number of colorants or primaries associated with the profile.

int gscms_get_pcs_channel_count(gcmmhprofile_t profile);

Get the channel count for the profile connection space. In general this will be three but could be larger for device link profiles. 


\section{ICC Color, the Display List and Multi-Threaded Rendering}

Ghostscript's display list is referred to as the c-list (command list). Using the option -dNumRenderingThreads $=X$, it is possible to have Ghostscript's c-list rendered with $X$ threads. In this case, each thread will simultaneously render different horizontal bands of the page. When a thread completes a band, it will move on to the next one that has not yet been started or completed by another thread. Since color transformations are computationally expensive, it makes sense to perform these operations during the multi-threaded rendering. To achieve this, ICC profiles can be stored in the c-list and the associated color data stored in the c-list in its original source space.

Vector colors are typically passed into the c-list in their destination color space, which is to say that they are already converted through the CMM. Images however are not necessarily pre-converted but are usually put into the c-list in their source color space. In this way, the more time consuming color conversions required for images occurs during the multi-threaded rendering phase of the c-list. Transparency buffers also require extensive color conversions. These buffers are created during the c-list rendering phase and will thus benefit from having their color conversions occur during the multi-threaded rendering process.

\section{$7 \quad$ PDF and PS CIE color space handling}

One feature of Ghostscript is that all color conversions can be handled by the external CMM. This enables more consistent specialized rendering based upon object type and rendering intents. Most CMMs cannot directly handle CIE color spaces defined in PostScript or the CalGray and CalRGB color spaces defined in PDF. Instead most CMMs are limited to handling only ICC-based color conversions. To enable the handling of the non ICC-based color spaces, Ghostscript converts these to equivalent ICC forms. The profiles are created by the functions in gsicc_create.c. Note that gsicc_create.c requires icc34.h, since it uses the type definitions in that file in creating the ICC profiles from the PS and PDF CIE color spaces.

PostScript color spaces can be quite complex, including functional mappings defined by programming procedures. Representing these operations can require a sampling of the 1-D procedures. Sampling of functions can be computationally expensive if the same non-ICC color space is repeatedly encountered. To address this issue, the equivalent ICC profiles are cached and a resource id is used to detect repeated color space settings within the source document when possible. The profiles are stored in the profile cache indicated in Figure 1. In PDF, it is possible to define CIELAB color values directly. The ICC profile lab.icc contained in iccprofiles of Figure 1 is used as the source ICC profile for color defined in this manner. 
Currently PostScript color rendering dictionaries (CRDs) are ignored. Instead, a device ICC profile should be used to define the color for the output device. There is currently an enhancement request to enable the option of converting CRDs to equivalent ICC profiles.

The use of the command line option -dUseCIEColor will result in document DeviceGray, DeviceRGB and DeviceCMYK source colors being substituted respectively by Postscript CIEA, CIEABC and CIEDEFG color spaces. In this case, -sDefaultGrayProfile, -sDefaultRGBProfile and -sDefaultCMYKProfile will not specify the ICC profiles to use for these source spaces. The PS color spaces that are used with-dUseCIEColor are defined in the directory gs/Resource/ColorSpace within the files DefaultGray, DefaultRGB and DefaultCMYK. Note that Ghostscript will end up converting these PS color spaces to equivalent ICC profiles using the methods in gsicc_create.c, so that the ICC-based CMM can perform the proper color conversions.

\section{DeviceN and Separation colors}

\subsection{Spot Colors}

Spot colors, which are sometimes referred to as named colors, are colorants that are different than the standard cyan, magenta, yellow or black colorants. Spot colors are commonly used in the printing of labels or for special corporate logos for example. In PostScript and PDF documents, color spaces associated with spot colors are referred to as separation color spaces. The ICC format defines a structure for managing spot colors called a named color profile. The structure consists of a table of names with associated CIELAB values for 100 percent tint coverage. In addition, the table can contain optional CMYK device values that can be used to print the same color as the spot color. In practice, these profiles are rarely used and instead the proofing of spot colors with CMYK colors is often achieved with proprietary mixing models. The color architecture of Ghostscript enables the specification of a structure that contains the data necessary for these mixing models. When a fill is to be made with a color in a separation color space, a call is made passing along the tint value, the spot color name and a pointer to the structure so that the proprietary function can return the device values to be used for that particular spot color. If the function cannot perform the mapping, then a NULL valued pointer is returned for the device values, in which case the alternate tint transform specified in the PDF or PS content is used to map the spot tint color.

\subsection{DeviceN Colors}

DeviceN color spaces are defined to be spaces consisting of a spot color combined with one or more additional colorants. A DeviceN color space can be handled in a similar proprietary fashion as spot colors if desired. The details of this implementation are given in Section 8.3 . 


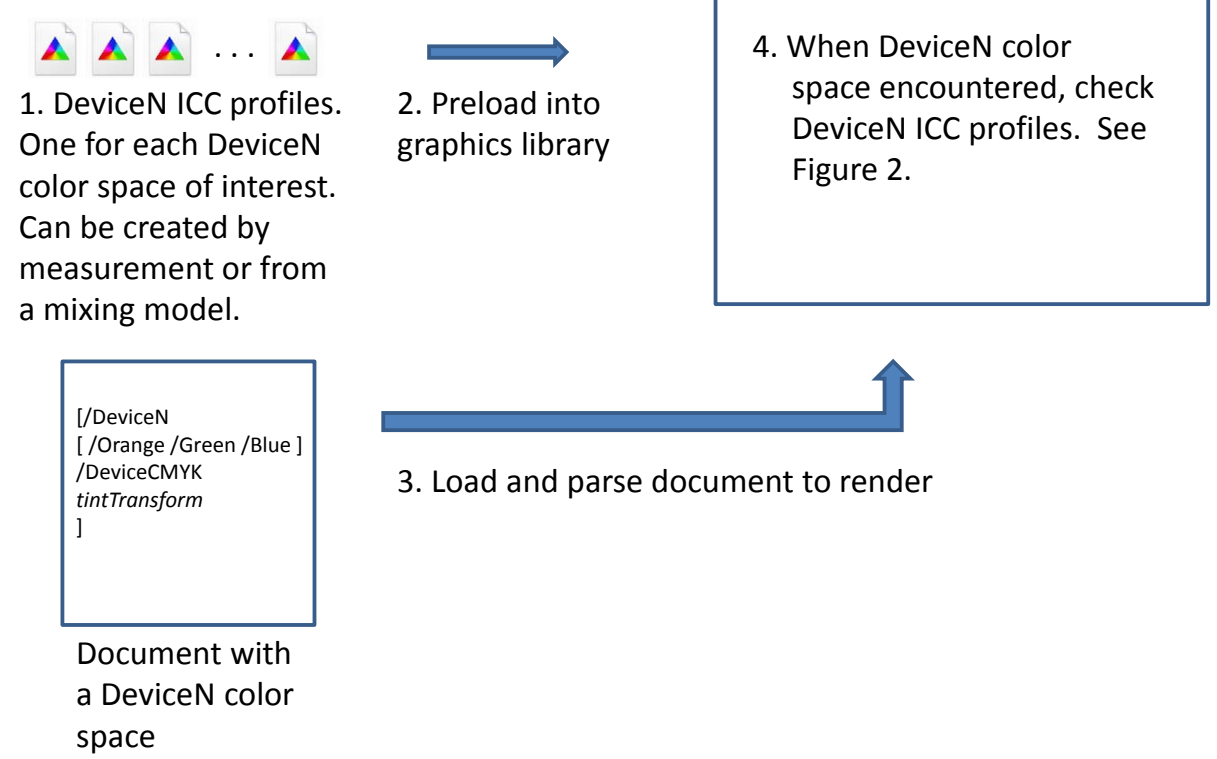

Figure 7: Flow for use of xCLR source profiles to define DeviceN color in PDF and PS source files

Ghostscript also provides an ICC-based approach for handling DeviceN source colors. In this approach, xCLR ICC source profiles can be provided to Ghostscript upon execution through the command line interface using -sDeviceNProfile. These profiles describe how to map from DeviceN tint values to CIELAB values. The profiles must include the colorantTableTag. This tag is used to indicate the colorant names and the lay-down order of the inks. The colorant names are associated with the colorant names in a DeviceN color space when it is encountered. If a match is found, the xCLR ICC profile will be used to characterize the source DeviceN colors. Note that the colorant orders specified by the names may be different in the source profile, necessitating the use of a permutation of the DeviceN tint values prior to color management. An overview of the process is shown in Figure 7. The directory ./gs/toolbin/color/icc_creator contains a Windows application for creating these DeviceN source ICC profiles. Refer to the README.txt file for details and for an example.

In Microsoft's XPS format, all input DeviceN and Separation type colors are required to have an associated ICC profile. If one is not provided, then per the XPS specification [4] a 
SWOP CMYK profile is assumed for the first four colorants and the remaining colorants are ignored. With PDF DeviceN or Separation colors, the document defines a tint transform and an alternate color space, which could be any of the CIE (e.g. CalGray, CalRGB, Lab, ICC) or device (e.g. Gray, RGB, CMYK) color spaces. If the input source document is PDF or PS and the output device does not understand the colorants defined in the DeviceN color space, then the colors will be transformed to the alternate color space and color managed from there assuming an external xCLR ICC profile was not specified as described above.

For cases when the device does understand the spot colorants of the DeviceN color space, the preferred handling of DeviceN varies. Many prefer to color manage the CMYK components with a defined CMYK profile, while the other spot colorants pass through unmolested. This is the default manner by which Ghostscript handles DeviceN input colors. In other words, if the device profile is set to a particular CMYK profile, and the output device is a separation device, which can handle all spot colors, then the CMYK process colorants will be color managed, but the other colorants will not be managed. If it is desired that the CMYK colorants not be altered also, it is possible to achieve this by having the source and destination ICC profiles the same. This will result in an identity transform for the CMYK colorants.

It should be noted that an ICC profile can define color spaces with up to 15 colorants. For a device that has 15 or fewer colorants, it is possible to provide an ICC profile for such a device. In this case, all the colorants will be color managed through the ICC profile. For cases beyond 15, the device will be doing direct printing of the DeviceN colors outside of the 15 colorants.

\subsection{DeviceN, Spot Color Customization and Direct Color Re- placement}

In earlier versions of Ghostscript, there existed a compile define named

CUSTOM_COLOR_CALLBACK, which provided developers with a method to intercept color conversions and provide customized processing in particular for Separation and DeviceN input color spaces. Using specialized mixing models in place of the standard tint transforms, accurate proofing of the spot colorants was obtainable. An interface for custom handling of separation colors and DeviceN is now performed by customization of the function gsicc_transform_named_color. An example, implementation is currently in place, which uses a look-up-table based upon the colorant name. The look-up-table is stored in the device_named object of the icc manager. The structure can be stored in the location using -sNamedProfile = c:/my_namedcolor_stucture. An example file is contained in gs/toolbin/color/named_color. The PDF file includes both DeviceN and separation colors and the named color structure contains the CIELAB values of the colorants. Mixing of the colors is performed with the sample function gsicc_transform_named_color. 
DeviceN color handling can also defined by an object stored in the device_n entry of the icc_manager. Currently, the example implementation is to use an array of ICC profiles that describe the mixing of the DeviceN colors of interest. This array of profiles is contained in the device_n entry of the icc_manager. In this case, a multi-dimensional look-up-table is essentially used to map the overlayed DeviceN colors to the output device colorants.

In addition to Custom DeviceN and Separation color handling, it is possible to force a path to a completely customizable color management solution for any object type using the -sSourceObjectICC = filename command line option and the "Replace" key word described above. In this case, all the RGB or CMYK source objects will be forced through the methods in gsicc_replacecm.c. All of the mapping is handled in the function gsicc_rcm_transform_general. Note that through the use of the settings in -sSourceObjectICC it is possible to have this flow occur only for certain source object types as well as for only those objects that are not already colorimetrically defined.

Note that the graphics library is unaware of what type of color management is being performed (i.e. the custom replace color (rc) method, none or ICC) but simply asks for a link and applies it to a color or buffer of colors. The mappings are performed through the procedures of Ghostscript's link structure. The procedure structure, which is a member variable of gsicc_link_t is defined in gscms.h and given as

typedef struct gscms_procs_s \{ gscms_trans_buffer_proc_t map_buffer; gscms_trans_color_proc_t map_color; gscms_link_free_proc_t free_link

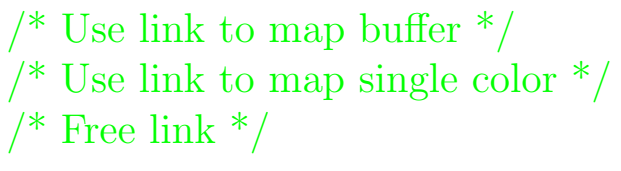
\} gscms_procs_t;

For the CMM that is interfaced with Ghostscript, these procedures are populated with map_buffer $=$ gscms_transform_color_buffer; map_color $=$ gscms_transform_color; free_link = gscms_release_link;

The unmanaged color option -dUseFastColor uses special mapping procedures where map_buffer $=$ gsicc_nocm_transform_color_buffer; map_color $=$ gsicc_nocm_transform_color; free_link = gsicc_nocm_freelink;

In this way, the fact that unmanaged color is occurring is opaque to Ghostscript. Similarly, the use of "Replace" in -sSourceObjectICC results in a link with the procedures 
map_buffer $=$ gsicc_rcm_transform_color_buffer;

map_color $=$ gsicc_rcm_transform_color;

free_link = gsicc_rcm_freelink;

This is provided as an example implementation for RIP OEMs desiring to provide unique color solutions for their products. Note that the file gsicc_nocm.c and gs_replacecm.c both use operators tied to the Link Cache to enable the links that are not ICC based to be stored in the same cache as the ICC based ones. If a developer wishes to implement their own color conversion methods and make use of Ghostscript's Link Cache they can do so following the examples in these files.

\section{PCL and XPS Support}

PCL [5] makes use of the new color management architecture primarily through the output device profiles as source colors are typically specified to be in the sRGB color space.

Full ICC support for XPS [4 is contained in ghostxps. This includes the handling of profiles for DeviceN color spaces, Named colors and for profiles embedded within images.

\section{References}

[1] Specification ICC.1:2004-10 (Profile version 4.2.0.0) Image technology colour management - Architecture, profile format, and data structure. (http://www.color.org/ICC1v42_2006-05.pdf), Oct. 2004.

[2] PostScript Language Reference Third Edition, Adobe Systems Incorporated, AddisonWesley Publishing, (http://partners.adobe.com/public/developer/ps/index_specs.html) Reading Massachusetts, 1999.

[3] PDF Reference Sixth Edition Ver. 1.7, Adobe Systems Incorporated, (http://www.adobe.com/devnet/pdf/pdf_reference.html), November 2006.

[4] XML Paper Specification Ver. 1.0, Microsoft Corporation, (http://www.microsoft.com/whdc/xps/xpsspec.mspx), 2006.

[5] PCL5 Printer Language Technical Reference Manual, Hewlett Packard, (http://h20000.www2.hp.com/bc/docs/support/SupportManual/bpl13210/bpl13210.pdf), October 1992. 
Copyright (c) 2017, Artifex Software Inc. All rights reserved. 